\title{
Violência no contexto escolar e ideação suicida na adolescência
}

\author{
Violence in the school context and suicidal ideation in adolescence \\ Violencia en el contexto escolar e ideación suicida en la adolescencia
}

\section{Maria Aparecida Beserra', Sinara de Lima Souza' ${ }^{\text {II }}$, Marta Angélica Iossi Silva ${ }^{I I I}$, Cláudia Alves de Sena ${ }^{\mathrm{IV}}$, Cristina Maria Mendes Resende ${ }^{\mathrm{V}}$, Maria das Graças Carvalho Ferriani ${ }^{\mathrm{VI}}$}

\begin{abstract}
Resumo: Objetivo: analisar a violência escolar sofrida e praticada e a sua associação com a ideação suicida, entre adolescentes com 12 a 18 anos. Método: pesquisa realizada com 643 adolescentes, matriculados em escolas estaduais da cidade do Recife, Pernambuco, Brasil. Foram aplicados dois questionários: "Global School-based Student Health Survey" e "Violência na Escola". Para análise estatística utilizou-se o teste Qui-quadrado, grau de associação foi analisado por meio da razão de prevalências. Resultados: as prevalências de violência escolar e de ideação suicida foram de $62,2 \%$ e $17,4 \%$, respectivamente. Cerca de $28 \%$ dos adolescentes relataram já terem se sentido muito triste, $14 \%$ deles declararam sentir solidão frequentemente. Houve associação significativa da ideação suicida com a violência sofrida na faixa etária de 15-18 anos. Conclusão: os resultados apontam para a necessidade de ações de saúde, que visem fortalecer os fatores de proteção e prevenção das situações de risco para a violência e ideação suicida.
\end{abstract}

Descritores: Violência; Estudantes; Comportamento do adolescente; Ideação suicida

\begin{abstract}
Objective: to analyze the school violence suffered and practiced and its association with suicidal ideation among adolescents aged 12 to 18 years. Method: study conducted with 643 adolescents registered in state schools in the city of Recife, Pernambuco, Brazil. Two questionnaires were applied: "Global School-based Student Health Survey" and "Violence at School". For statistical analysis, the Chi-square test was used, degree of association was analyzed using the prevalence ratio. Results: the prevalence of school violence and suicidal ideation was $62.2 \%$ and $17.4 \%$, respectively. About $28 \%$ of the adolescents reported having felt very sad, $14 \%$ of them reported feeling loneliness frequently. There was a significant association between suicidal ideation and violence in
\end{abstract}

\footnotetext{
${ }^{\text {I }}$ Enfermeira. Professora Doutora da Universidade de Pernambuco. Recife, Pernambuco, Brasil. E-mail: aparecida.beserra@upe.br, Orcid: https://orcid.org/0000-0002-5315-5589

II Enfermeira. Professora Doutora da Universidade Estadual de Feira de Santana. Feira de Santana, Bahia, Brasil. E-mail: sinarals@uefs.br, Orcid: https://orcid.org/0000-0001-8003-2093

III Enfermeira. Professora Doutora da Universidade de São Paulo, Ribeirão Preto. São Paulo, Brasil. E-mail: maiossi@eerp.usp.br, Orcid: https://orcid.org/0000-0002-9967-8158

IV Enfermeira. Professora Doutora da Universidade de Pernambuco. Recife, Pernambuco, Brasil. E-mail: claudia.sena@upe.br, Orcid: https://orcid.org/0000-0003-1162-3601

v Nutricionista. Pós-doutoranda da Universidade de Pernambuco. Recife, Pernambuco, Brasil. E-mail: cmariamendesr@gmail.com, Orcid: https://orcid.org/0000-0002-2895-8558

VI Enfermeira. Professora Doutora da Universidade de São Paulo. Ribeirão Preto, São Paulo, Brasil. E-mail: gracacarvalho@usp.br, Orcid: https://orcid.org/0000-0002-7103-4895
} 
the age group of 15-18 years. Conclusion: the results point to the need for health actions aimed at strengthening the protective and prevention factors of risk situations for violence and suicidal ideation.

Descriptors: Violence; Students; Adolescent behavior; Suicidal ideation

Resumen: Objetivo: analizar la violencia escolar sufrida y practicada y su asociación con la ideación suicida entre adolescentes de 12 a 18 años. Método: estudio realizado con 643 adolescentes matriculados en escuelas estatales en la ciudad de Recife, Pernambuco, Brasil. Se aplicaron dos cuestionarios: "Global School-based Student Health Survey" y "Violencia en la Escuela". Para el análisis estadístico, se utilizó la prueba Chi-cuadrada, se analizó el grado de asociación utilizando la relación de prevalencia. Resultados: la prevalencia de la violencia escolar y la ideación suicida fue del $62,2 \%$ y del $17,4 \%$, respectivamente. Alrededor del $28 \%$ de los adolescentes informaron que se habían sentido muy triste, el $14 \%$ de ellos reportaron sentir la soledad con frecuencia. Hubo una asociación significativa entre la ideación suicida y la violencia en el grupo de edad de 15-18 años. Conclusión: los resultados apuntan a la necesidad de acciones sanitarias encaminadas a fortalecer los factores de protección y prevención de las situaciones de riesgo para la violencia y la ideación suicida.

Descriptores: Violencia; Estudiantes; Conducta del adolescente; Ideación suicida

\section{Introdução}

A violência é um problema social grave que atinge diferentes populações e precisa ser estudada transversalmente. Esta temática complexa e abrangente tem integrado a pauta de discussões e preocupações no Brasil e em vários países, principalmente, quando a sua ocorrência está relacionada ao ambiente escolar. Segundo Fundo das Nações Unidas para a Infância (UNICEF) metade dos adolescentes no mundo são vítimas de violência na escolar. ${ }^{1}$ Esse fenômeno não pode ser sustentado e banalizado pela cotidianidade, principalmente quando ocorre em um ambiente como a escola, que é uma das instituições responsáveis pela formação psicossocial das crianças e dos adolescentes. Baseado no entendimento de que a violência na escola traz prejuízos à saúde de todos os indivíduos envolvidos, especialmente das crianças e dos adolescentes, o presente artigo incidiu pela investigação dentro do contexto da saúde pública, tendo como foco o adolescente.

O desenvolvimento do adolescente envolve uma progressiva independência e autonomia da família, uma maior associação com os pares, a formação da identidade e a maturação fisiológica e cognitiva. Evidências apontam para a associação entre a entrada precoce na 
puberdade e a adoção de comportamentos de risco para a saúde, além de aumento na exposição a esses fatores com o avançar da idade. ${ }^{2}$ Outrossim, comportamentos, de saúde ou de risco à saúde, adquiridos na adolescência tendem a se perpetuar na vida adulta, com as respectivas consequências para a qualidade de vida. ${ }^{3}$ Nesse sentido, ressalta-se a importância da violência escolar entre adolescentes ser analisada de modo que se explore os problemas de comportamento que venha comprometer a sua integridade física e mental, à exemplo, a ideação suicida.

A ideação suicida é um grave problema de saúde pública, sendo considerada um fator predisponente para a violência autoinfligida. Durante décadas, tem sido uma das principais causas de morte entre adolescentes na população mundial.4-5 Segundo o relatório da Organização Mundial da Saúde (OMS) de 2014, o suicídio é a terceira principal causa de morte de jovens de 15 a 24 anos, e a sexta causa de morte de crianças de 5 a 14 anos. ${ }^{3}$ No Brasil, entre 2002 e 2012, a taxa anual de suicídio entre adolescentes e jovens aumentou em 15,3\%, passando de 2.515 para 2.900 mortes. Levando-se em conta a população com idade igual ou superior a 15 anos, a faixa etária de 15 a 19 anos foi a que apresentou maior crescimento do número de casos de suicídio no período de 1990 a $2012 .^{6}$

Em geral, as pessoas que vêm a cometer suicídio, passaram por um processo longo e complexo de ideação suicida, planejamento e tentativa. ${ }^{7}$ Os dados da Pesquisa Nacional da Saúde do Escolar de 2015 revelaram que os escolares se envolveram em brigas e sofreram agressão física, tanto no âmbito escolar, como no familiar, dentre as consequências a médio e longo prazo de tais situações pode-se citar maior risco de desenvolver transtornos emocionais como ansiedade, depressão, transtornos alimentares, abuso de drogas, ideação suicida e até o suicídio. ${ }^{2}$ Destaque que a ideação suicida prediz o ato e, por isso, torna-se imprescindível não só detectá-la precocemente, mas também ampliar o entendimento sobre a sua ocorrência na adolescência e a sua relação com outros fenômenos que podem ocorrer nesse momento do ciclo vital, como a violência escolar. A avaliação de fatores de risco para a saúde é uma atividade 
importante tanto para o desenvolvimento de programas preventivos quanto para a atuação no contexto da intervenção, como nos casos de violência, abuso, autoagressão, suicídio, entre outros problemas relevantes do nosso tempo. ${ }^{7}$

Nesse contexto, objetivou-se analisar a violência escolar sofrida e praticada e a sua associação com a ideação suicida, entre adolescentes com 12 a 18 anos.

\section{Método}

Este artigo faz parte de um estudo realizado com o objetivo de analisar a violência sofrida e/ou praticada por adolescentes no contexto escolar e os fatores individuais e ambientais associados, em escolas públicas da cidade do Recife, Pernambuco, Brasil. ${ }^{8}$ Trata-se de um delineamento transversal em uma população estimada em 4.905 estudantes matriculados em seis escolas estaduais do bairro de Santo Amaro, na cidade do Recife.

O bairro de Santo Amaro integra a 1ª Região Político-Administrativa do Recife, Centro da cidade, formada por um total de onze bairros. ${ }^{9}$ Durante muitos anos, este bairro foi considerado o mais violento da cidade. A implantação do programa "Pacto pela Vida" reduziu significativamente o índice de criminalidade neste bairro, mas o tráfico de drogas ainda se faz muito presente, aumentando a sensação de insegurança dos moradores e frequentadores do local..$^{9}$ A escolha desse bairro como campo de estudo foi baseada não só nessas características, mas também pelo fato da Universidade de Pernambuco encontrar-se localizada nele. A proximidade da universidade com a comunidade local favorece o desenvolvimento de trabalhos de extensão universitária e de pesquisa pelos estudantes de graduação e pós-graduação, com o objetivo central de subsidiar ações futuras de promoção da saúde dos escolares.

A amostra foi composta por 643 adolescentes, com faixa etária entre 12 e 18 anos, conforme preconizado pelo Estatuto da Criança e Adolescente ${ }^{10}$ matriculados nos Ensinos Fundamental e Médio. Para o tamanho amostral e seleção dos indivíduos, utilizou-se o método 
5 | Beserra MA, Souza SL, Silva MAI, Sena CA, Resende CMM, Ferriani MGC

de amostragem probabilística estratificada por escola, considerando o tamanho populacional estimado de 4.905 estudantes matriculados nas seis escolas estaduais do bairro estudado; a margem de erro de 5\%; a confiabilidade de $95 \%$ de que a margem de erro não seja ultrapassada; a proporção esperada de $50 \%$ para cada categoria de resposta, valor este que maximiza o tamanho amostral. O número total de estudantes da amostra (643) foi distribuído proporcionalmente entre as seis escolas selecionadas e nos três turnos letivos. Todos os cálculos para determinação da amostra foram realizados por meio do programa Epi-Info versão 6.04d para DOS. Foi realizado sorteio aleatório por turno e turma nas escolas (extratos) participantes do estudo.

Como critérios de inclusão: adolescentes de ambos os sexos, na faixa etária de 12 a 18 anos cujos pais ou responsáveis assinaram o termo de consentimento livre e esclarecido e o termo de assentimento assinado pelo adolescente. Foram excluídos os adolescentes que apresentaram dificuldades em responder o questionário devido algum déficit cognitivo, que estavam de licença maternidade, ou com suspensão de suas atividades escolares.

O procedimento de coleta de dados foi organizado em fases e momentos de encontros, e distribuídos da seguinte forma: $1^{\circ}$ encontro: Apresentação do projeto para os pais, mães e responsáveis na reunião mensal realizada pelas escolas. $2^{\text {o }}$ encontro: Apresentação do projeto para as turmas do ensino fundamental e médio. Realização de seleção aleatória dos participantes de acordo com os critérios de inclusão e exclusão, e distribuição dos termos de consentimento e assentimento. De posse dos termos assinados procedeu-se com o $3^{\mathbf{0}}$ encontro: Aplicação dos questionários com duração máxima do tempo de uma aula (equivalente a 45 minutos).

Os dados foram coletados no período de maio a outubro de 2014, utilizando-se dois questionários (já validados no Brasil) autoaplicáveis: o primeiro, denominado Global SchoolBased Student Health Survey, ${ }^{11}$ continha questões sociodemográficas e comportamentais, o segundo continha questões relacionadas à violência sofrida e/ou praticada dentro do contexto 
escolar. ${ }^{12}$ Os questionários foram digitalizados em planilhas eletrônicas, e os dados foram analisados no programa SPSS versão 21.

As variáveis selecionadas para análise foram classificadas em: i) protagonismo do adolescente enquanto vítima e agressor da violência no contexto escolar (tipo de violência, quem foi o agressor, quem foi a vítima, o lugar onde ocorreu a violência, quantas vezes foi vítima ou agressor, se continua sendo agredido ou agredindo); ii) sentimentos relacionados (solidão, tristeza, quantos amigos, sono, religião, experiência na escola e em casa); e iii) comportamento de risco para a saúde (ideação suicida).

As demais variáveis independentes: Indicadores sociodemográficos foram: Sexo (Masculino/feminino), faixa etária de (12 a 14 anos, 15 a 18 anos); escolaridade da mãe $(<8$ anos de estudo, > 8 anos de estudo); religião (com religião, sem religião); escolaridade (ensino fundamental, ensino médio); estado conjugal (solteiro, casado); local da residência (rural, urbana); mora com quem (mãe, pai, avós, pai e mãe).

Para análise dos dados foi construído um banco de dados no programa SPSS, versão 21. Para a análise foram utilizados aspectos descritivos por meio das frequências (absoluta e relativa), a existência de associação entre duas variáveis categóricas foi avaliada utilizando-se o teste estatístico Qui-quadrado ou o teste Exato de Fisher, adotando-se a margem de erro de 5\%, e o grau de associação entre as variáveis foi avaliado pela razão de prevalências e o respectivo intervalo de confiança (IC95\%). O projeto foi aprovado pelo Comitê de Ética em Pesquisa da Universidade de Pernambuco e seguiu os preceitos éticos da Resolução 466/12, do Conselho Nacional da Saúde registrado sob nº 705.598 em 01/07/2014.

\section{Resultados}

As principais características da amostra de 643 adolescentes pesquisados foram: predominância na faixa etária de 15 a 18 anos (56,5\%) em relação à faixa etária de 12 a 14 anos 
(43,5\%), predominância do sexo feminino (64,2\%), solteiros(as) $(93,6 \%)$, que não trabalhavam $(87,9 \%)$, e se consideravam não brancos $(82,3 \%)$, principalmente pardo/mulato/moreno (70,6\%). No quesito escolaridade, $54,1 \%$ dos adolescentes cursavam o ensino médio, e 52,3\% das mães dos adolescentes pesquisados tinham de 8 a 11 anos de escolaridade. Quanto à moradia, a maioria residia na região urbana $(95,5 \%)$, em companhia da mãe $(89,7 \%)$, e pouco mais da metade deles $(56,8 \%)$ residia com o pai. Em relação à religiosidade, $56,6 \%$ dos adolescentes se consideraram praticantes da sua religião, havendo predominância de evangélicos (40,4\%) e católicos (29,5\%).

A maioria dos participantes $(62,2 \%)$ do estudo revelou ter se sentido vítimas de agressão por parte de colegas ou de outras pessoas da escola, 51,9\% referiu ter perpetrado a violência nas duas semanas que antecederam a realização da pesquisa (Tabela 1 e 2). O tipo de agressão relatado com maior frequência na qualidade de vítima e agressor foi a verbal $(54,2 \% ; 55,6 \%)$ respectivamente, sendo que a maioria agredida apenas uma vez $(79,8 \%)$, por uma pessoa $(65,5 \%)$, no entanto, cerca de $12,5 \%$ continuaram sendo agredidos pelas mesmas pessoas. Quase a totalidade dos agressores eram colegas de sala (96\%), e o local da escola onde ocorreram os atos de violência mais citado foi a sala de aula $(45,9 \%)$. Cerca de metade $(51,3 \%)$ dos casos de agressão foi presenciada por terceiros, os quais, em sua maioria, não tomaram atitude alguma (36,7\%) ou apoiaram o adolescente vítima de violência e aconselharam-no a afastar-se do agressor $(27,1 \%)$, na violência perpetrada $26,3 \%$ referiu que foi apoiado pelos colegas que sorriram da situação (Tabela 1 e 2).

Tabela 1 - Distribuição dos adolescentes escolares, segundo as questões relacionadas ao fato de terem sido vítimas de violência na escola. Recife, Pernambuco, Brasil, 2013.

\begin{tabular}{lcc}
\hline Variável & N & $\mathbf{\%}$ \\
\hline Nas duas últimas semanas, alguma vez você se sentiu vítima de alguma & & \\
agressão, por parte de colegas ou de outras pessoas da escola? ${ }^{\text {(a) }}$ & & \\
Sim & 400 & 62,2 \\
Não & 243 & 37,8 \\
Tipos de agressão sofridas (b) & & \\
Física & 129 & 24,0 \\
$\quad$ Verbal & 292 & 54,2
\end{tabular}


Psicológica

Patrimonial

Sexual

Outras agressões

Quantas vezes você foi agredido ou perseguido? ${ }^{(c)}$

Uma vez

Duas ou mais vezes

Em qual local da escola ocorreram essas situações? ${ }^{(d)}$

Sala de aula

Pátio

Corredores e/ou escadas

Refeitório

Espaço de Educação Física

Banheiro

Por quantas pessoas você foi agredido? ${ }^{(c)}$

Uma

Duas

Três ou mais

Por quais pessoas você foi agredido? ${ }^{(e)}$

Colegas de sala

Professor/Diretor/ outras Pessoas da área

Você ainda continua sendo agredido/perseguido por essas pessoas? ${ }^{\text {(c) }}$

Sim

Não

Alguém presenciou essas situações? ${ }^{(c)}$

Sim

Não

O que fizeram as pessoas que presenciaram? ${ }^{(\mathfrak{f})}$

Não fez nada

Recorreu a um adulto

Pediu ao agressor para parar

Apoiou o agressor/ Sorriu da situação

Apoiou você/ Aconselhou você a se afastar do agressor
$44 \quad 8,2$

$26 \quad 4,8$

$26 \quad 4,8$

$22 \quad 4,1$

$319 \quad 79,8$

$81 \quad 20,2$

$224 \quad 45,9$

$83 \quad 17,0$

$88 \quad 18,0$

$26 \quad 5,3$

$11 \quad 2,2$

$18 \quad 3,7$

$262 \quad 65,5$

$94 \quad 23,5$

$44 \quad 11,0$

38496,2

$19 \quad 4,8$

$50 \quad 12,5$

$350 \quad 87,5$

$205 \quad 51,3$

19548,8

$88 \quad 36,7$

$19 \quad 7,9$

$25 \quad 10,4$

$30 \quad 12,5$

$65 \quad 27,1$

Nota: (a), (c): Percentuais calculados em relação ao total de respostas, sendo (a) 643; (c) 400.(b), (d), (e), (f):

Considerando-se que um mesmo entrevistado tenha citado mais de uma opção de resposta, registrou-se a base para o cálculo dos percentuais e não o total de respostas, sendo (b) 539; (d) 488; (e) 403; (f) 240.

Tabela 2 - Distribuição dos adolescentes escolares, segundo as questões relacionadas ao fato de terem sido os autores do(s) ato(s) de violência na escola. Recife - Pernambuco, 2013.

\begin{tabular}{lll}
\hline Variável & $\mathbf{N}$ & $\%$ \\
\hline
\end{tabular}

Nas duas últimas semanas, alguma vez você teve atitudes agressivas contra alguém (estudante, professor ou funcionário da escola)? ${ }^{\text {(a) }}$ 
$\operatorname{Sim}$

Não

Quais tipos de atitudes agressivas você teve? ${ }^{(b)}$

Física

$130 \quad 33,5$

Verbal

21655,6

Psicológica

$20 \quad 5,1$

Patrimonial

$10 \quad 2,6$

Sexual

$6 \quad 1,5$

Local da escola ocorreram essas situações? ${ }^{(c)}$

Sala de aula

$195 \quad 49,7$

Pátio

Corredores e/ou escadas

$66 \quad 16,8$

Refeitório

6616,8

Banheiro

$18 \quad 4,6$

$17 \quad 4,3$

Quantas vezes você teve essa atitude? ${ }^{\text {(d) }}$

Uma

$260 \quad 77,8$

Duas

$35 \quad 10,5$

Três ou mais

$39 \quad 11,7$

Você praticou em grupo ou sozinho? ${ }^{(d)}$

Sozinho

$217 \quad 65,0$

Em grupo

11735,0

Você agrediu ou perseguiu a mesma pessoa mais de uma vez? ${ }^{(d)}$

Sim

$23 \quad 6,9$

Não

31193,1

Quais as razões deste comportamento? ${ }^{(e)}$

Vingança

$57 \quad 15,2$

Defesa de outros colegas

$32 \quad 8,5$

Brincadeira

$168 \quad 44,8$

Reação a provocadores

$32 \quad 8,5$

Irritação

Quem eram essas pessoas? ${ }^{(\mathrm{f})}$

Colega de sala

$319 \quad 94,37$

Professor (es)/Outro funcionário/ Diretor

$19 \quad 5,7$

Alguém presenciou essas situações? ${ }^{(\mathrm{d})}$

Sim

$179 \quad 53,6$

Não

15546,4

O que fizeram as pessoas que presenciaram? ${ }^{(\mathrm{g})}$

Não fez nada

$92 \quad 47,4$

Recorreu a um adulto

$10 \quad 5,1$

Pediu ao agressor para parar

$15 \quad 7,7$

Apoiou o agredido

$14 \quad 7,2$

Apoiou o agressor/ Sorriu da situação

5126,3

Você gostaria de ter um comportamento diferente com essas pessoas? ${ }^{(d)}$

$\operatorname{Sim}$

Não

$149 \quad 44,6$


O que você já fez para mudar esse comportamento? ${ }^{(h)}$

$\begin{array}{lll}\text { Não quero mudar o meu comportamento } & 99 & 28,4\end{array}$

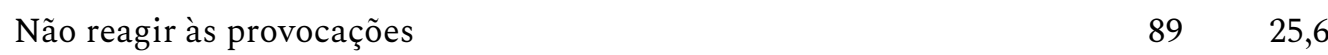

$\begin{array}{lll}\text { Controlar-me melhor } & 86 & 24,7\end{array}$

$\begin{array}{lrr}\text { Conviver mais com os colegas } & 48 & 13,8\end{array}$

Nota: (a), (c), (d): Percentuais calculados em relação ao total de respostas, sendo (a) 643; (c) 392; (d) 334. (b), (e)-(h): Considerando-se que um mesmo entrevistado tenha citado mais de uma opção de resposta, registrou-se a base para o cálculo dos percentuais e não o total de respostas, sendo (b) 388; (e) 375 ; (f) $338 ;$ (g) 194; (h) 348.

Em relação aos sentimentos predominantes nos últimos doze meses anteriores à realização da pesquisa, a maioria dos adolescentes $(68,9 \%)$ relatou já ter sentido solidão (Tabela 3). Quase um terço $(28,8 \%)$ dos adolescentes já havia se sentido muito triste ou sem esperança quase todos os dias ou durante duas semanas ou mais seguidas, a ponto de ter que interromper as suas atividades normais. Uma parcela dos adolescentes declarou ter sentido solidão na maioria das vezes ou sempre (14\%), já ter pensado seriamente em tentar suicídio $(17,4 \%)$, e inclusive ter feito planos sobre como tentaria se suicidar (14\%) (Tabela 3).

Tabela 3 - Distribuição absoluta e relativa da população de adolescentes escolares, segundo as questões relacionadas aos sentimentos e à ideação suicida. Recife, Pernambuco, Brasil, 2013.

\begin{tabular}{lcc}
\hline Variável & $\mathbf{N}$ & $\mathbf{\%}^{\text {(a) }}$ \\
\hline Durante os últimos 12 meses, quantas vezes você se sentiu sozinho? & 200 & 31,1 \\
Nunca & 353 & 54,9 \\
Raramente/ Algumas vezes & 90 & 14,0 \\
Na maioria das vezes/ Sempre & \\
Durante os últimos 12 meses, você se sentiu muito triste ou sem esperança quase todos & \\
os dias ou durante duas semanas ou mais seguidas, a ponto de interromper suas & \\
atividades normais? & 185 & 28,8 \\
Sim & 458 & 71,2 \\
Não & & \\
Durante os últimos 12 meses, você já pensou seriamente em tentar suicídio? & 112 & 17,4 \\
Sim & 531 & 82,6 \\
Não & & \\
Durante os últimos 12 meses, você fez planos sobre como tentaria se suicidar? & 90 & 14,0 \\
Sim & 553 & 86,0 \\
Não &
\end{tabular}

Nota: (a) Percentuais calculados com base no total de respostas $=643$. 
A análise bivariada de correlação entre a ideação suicida e violência sofrida e praticada pelos adolescentes (Tabela 4) revelou a existência de associação significativa da ideação suicida com a ocorrência de violência sofrida, não houve associação significativa entre a ideação suicida e a ocorrência de violência praticada.

Tabela 4 - Análise bivariada e razão de prevalência bruta (RP) da ideação suicida entre adolescentes escolares, segundo a violência sofrida e praticada pelos mesmos. Recife, Pernambuco, Brasil, 2013.

\begin{tabular}{|c|c|c|c|c|c|c|c|c|c|c|c|c|c|c|}
\hline \multirow{3}{*}{ Variável } & \multirow{2}{*}{\multicolumn{2}{|c|}{ Total }} & \multicolumn{6}{|c|}{ Violência sofrida } & \multicolumn{6}{|c|}{ Violência praticada } \\
\hline & & & \multicolumn{2}{|c|}{ Não } & \multicolumn{2}{|c|}{ Sim } & \multirow{2}{*}{$\begin{array}{l}\text { Valor } \\
\text { de } \mathrm{p}^{(\mathrm{a})}\end{array}$} & \multirow{2}{*}{$\frac{\mathrm{RP}}{\text { (IC 95\%) }}$} & \multicolumn{2}{|c|}{ Não } & \multicolumn{2}{|c|}{ Sim } & \multirow{2}{*}{$\begin{array}{l}\text { Valor } \\
\text { de } \mathrm{p}^{(\mathrm{a})}\end{array}$} & \multirow{2}{*}{$\begin{array}{c}\text { RP } \\
\text { (IC 95\%) }\end{array}$} \\
\hline & $\mathrm{n}$ & $\%$ & $\mathrm{n}$ & $\%$ & $\mathrm{n}$ & $\%$ & & & $\mathrm{n}$ & $\%$ & $\mathrm{n}$ & $\%$ & & \\
\hline \multicolumn{15}{|c|}{ Ideação suicida } \\
\hline Sim & 112 & 17,4 & 79 & 70,5 & 33 & 29,5 & $0,045^{\star}$ & $\begin{array}{c}1,17 \\
(1,02-1,34)\end{array}$ & 59 & 52,7 & 53 & 47,3 & 0,864 & $\begin{array}{c}1,02 \\
(0,84-1,23)\end{array}$ \\
\hline Não & 531 & 82,6 & 321 & 60,5 & 210 & 39,5 & & 1,00 & 275 & 51,8 & 256 & 48,2 & & 1,00 \\
\hline Total & 643 & 100 & 400 & 62,2 & 243 & 33,0 & & & 334 & 51,9 & 309 & 48,1 & & \\
\hline
\end{tabular}

Nota: (a) Valor de $p$ determinado por meio do teste Qui-quadrado de Pearson. ${ }^{*}$ Diferença significativa de $5 \%$. IC: intervalo de confiança.

\section{Discussão}

A prevalência da violência no contexto escolar, na condição em que os adolescentes foram vítimas das agressões, foi alta e atingiu $62,2 \%$ da amostra em estudo. Dado convergente aos relatos da literatura especializada nacional e internacional que apontam para uma prevalência estimada em $18 \%$ a $70 \%$ no Brasil ${ }^{13-14}$ e em $8 \%$ a $60 \%$ em outros países. ${ }^{15-16}$ Apesar das margens de diferença da prevalência da violência no contexto escolar, esses estudos ressaltam questões centrais em comum a serem consideradas: o espaço da escola não está imune à presença da violência, e a exposição dos adolescentes à violência escolar é uma preocupação mundial que tem levado os estudiosos de várias áreas de conhecimento a investigarem a ocorrência desse fenômeno, à exemplo da saúde e da educação. ${ }^{13-16}$ No contexto da área investigada e considerando-se que o campo de estudo se situa em um bairro com alta incidência 
de violência, nota-se que o entorno da escola e fatores de ordem cultural, relacional e econômico, podem influenciar os comportamentos violentos dos adolescentes pesquisados.

Em relação à violência praticada pelos adolescentes no ambiente escolar foi de (51,9\%), dois fatos preocupantes identificados foram à elevada incidência (44,6\%) de adolescentes agressores que afirmaram não querer mudar os seus comportamentos violentos e o percentual de $26,3 \%$ dos participantes do estudo que ao presenciar a agressão, apoiaram o agressor e acharam graça da situação. Tais comportamentos dos estudantes que presenciam a violência podem ser motivados pelo desejo de aumentar o seus status perante os pares, já que geralmente os agressores possuem maior popularidade, ou por medo, no intuito de autoproteção. É importante observar os comportamentos dos adolescentes que presenciam a atos violentos de seus pares, que têm sido negligenciados pelos estudiosos; o apoio a uma cultura de paz pode atuar como fator de proteção à violência entre adolescentes. ${ }^{17-18}$

Os principais protagonistas da violência foram os colegas de sala (96,2\%). De forma geral, os episódios de violência na escola que envolve apenas os estudantes são os que ocorrem com maior frequência e têm maior visibilidade para os diferentes atores desse contexto. Segundo a UNICEF no mundo, cerca de 150 milhões de estudantes entre 13 e 15 anos de idade já foram vítimas de violência por parte de seus colegas. Episódios de agressão aconteceram dentro e fora do ambiente escolar. ${ }^{1} \mathrm{O}$ fracasso escolar e a imposição de regras têm sido fatores explicativos da violência nas escolas; a construção de um ambiente escolar positivo e sustentável pode promover mudanças no comportamento dos estudantes e no processo de desenvolvimento. ${ }^{19}$

Os atos violentos ocorreram predominantemente na sala de aula $(45,9 \%)$, corroborando com outro estudo. ${ }^{13}$ A sala de aula é um dos contextos de maior influência sobre o clima geral do estabelecimento e a integração dos alunos, podendo ser lugar de discriminação e humilhação quotidiana por parte dos alunos e dos docentes. Tais implicações sobre as perspectivas que os professores têm a respeito de seus estudantes, influenciam na qualidade das relações que os 
docentes estabelecem em sala de aula em diversas situações, desde a atenção concedida, a própria dinâmica da aula (como é planejada e executada), como também as oportunidades oferecidas de protagonismo estudantil. ${ }^{19}$

No presente estudo, $14,0 \%$ dos adolescentes declararam sentir solidão frequentemente. Este resultado é semelhante ao obtido na Pesquisa Nacional de Saúde do Escolar realizada no ano de 2015, na qual 16,4\% dos estudantes do $9^{9}$ ano do ensino fundamental no Brasil declararam ter se sentido sozinhos nos doze meses que antecederam à pesquisa. ${ }^{2}$ Vale salientar que, no presente estudo, um percentual significativo de adolescentes $(28,8 \%)$ já se sentiu muito triste ou sem esperança quase todos os dias ou durante duas semanas ou mais seguidas, a ponto de interromper as suas atividades normais, 17,4\% pensaram seriamente em tentar o suicídio, e $14,0 \%$ fizeram planos sobre a forma de como se suicidar. O isolamento social, a carência de relações de amizade e a impossibilidade de idealizar o futuro, ser vítima de bullying, são fatores que podem causar a ideação e a execução de um comportamento suicidário. ${ }^{20-21}$

Em um estudo mais recente, foi constatado que $22,5 \%$ e $24,03 \%$ dos estudantes de 10 a 19 anos tem pensado e planejado suicídio, respectivamente, sentir-se sem esperança ou triste nos últimos 12 meses aumentou as chances de tentativa de suicídio em 4,5 [AOR = 4,51, IC 95\% (2,24, 9,08)] vezes. Também foi observado que os adolescentes que moram sozinhos têm cerca de duas vezes mais chances de relatos do que aqueles que moram com suas famílias, a convivência com familiares e/ou colegas encoraja os adolescentes a compartilhar seus sentimentos e pensamentos, especialmente durante eventos estressantes, o que poderia reduzir os distúrbios emocionais ou mentais, o apoio dos pais, a supervisão, a compreensão dos problemas e preocupações dos adolescentes e o apoio dos pares nas escolas são reconhecidos como fatores de proteção contra a ideação suicida. ${ }^{22}$

Foi verificado associação significativa da ideação suicida com a violência sofrida, corroborando com um estudo de meta-análise no qual, indicou que o envolvimento em violência 
escolar do tipo bullying está associado a ideação e comportamento suicida e ser vítima de intimidação está associada com maior risco. ${ }^{23}$ Ressalta-se que a presença de ideação suicida pode caracterizar um primeiro passo para a consolidação do suicídio. Portanto, desvelar os comportamentos associados à ideação suicida, como por exemplo, a violência sofrida e praticada no ambiente escolar é salutar, para que se possam identificar os determinantes comuns entre os diferentes tipos de comportamentos e, com base nesses determinantes, traçar ações de prevenção dos danos e de promoção da saúde. As escolas têm um papel único e importante a desempenhar na abordagem dos comportamentos relacionados à saúde de todos os estudantes. ${ }^{24}$ Programas de prevenção bem planejados e bem implementados, baseados na escola, podem reduzir significativamente os comportamentos de risco à saúde dos estudantes. ${ }^{4}$

Trabalhar as relações estabelecidas no espaço escolar favorece o processo ensinoaprendizagem, que poderá contribuir para a construção de um pensamento crítico e reflexivo dos adolescentes nas suas práticas diárias. A necessidade de se elaborar ações que visem à prevenção da violência e à promoção da saúde do escolar é emergente. Deste modo, um dos profissionais de saúde que possui papel fundamental na prevenção e combate deste tipo de agravo é o da enfermagem, na medida em que atua na promoção da saúde do escolar. ${ }^{25}$ Portanto, a articulação do enfermeiro com a escola é imprescindível para a execução de ações que visem à assistência na perspectiva da integralidade dos adolescentes, estimulando uma cultura de paz.

\section{Conclusão}

Nos resultados observa-se uma prevalência elevada, tanto para a violência sofrida quanto para a violência praticada, porém, na análise de associação da violência com a ideação suicida, encontrou-se associação desse evento apenas com a violência sofrida.

A principal limitação deste trabalho refere-se à análise de dados transversais, o que não permite com precisão, estabelecer relação causal da violência escolar com a ideação suicida 
entre adolescentes. Para estabelecer conexões causais mais precisas entre estas duas variáveis, são necessários estudos longitudinais para melhor compreensão temporal dessas associações. A utilização do autorrelato como medida para avaliar a vitimização e a ideação suicida também pode limitar a análise dos resultados, uma vez que exclui outros métodos, tais como relatos dos pais, professores e colegas, que contribuem para melhorar as medidas dos eventos e introduzem a possibilidade de variância de método compartilhado.

Em síntese, os dados encontrados evidenciam a importância da identificação dos fatores que estão diretamente associados com a violência sofrida e/ou praticada no ambiente escolar e a ideação suicida, que são essenciais para a construção do conhecimento sobre violência e suicidabilidade na adolescência. Apesar das limitações metodológicas, os resultados obtidos trazem à reflexão o quanto a violência escolar poderá aumentar o risco de suicídio entre adolescentes, com base em uma primeira explicação empiricamente apoiada, além de sinalizar para a necessidade da implantação e implementação de ações de saúde, que fortaleçam os fatores de proteção.

\section{Referências}

1. Fundo das Nações Unidas para a Infância (UNICEF). Metade dos adolescentes no mundo são vítimas de violência na escola [Internet]. 2018 [acesso em 2019 maio 29]. Disponível em: https://nacoesunidas.org/unicefmetade-dos-adolescentes-no-mundo-sao-vitimas-de-violencia-na-escola/

2. Instituto Brasileiro de Geografia e Estatística (IBGE). Pesquisa Nacional de Saúde do Escolar: 2015 [Internet]. Rio de Janeiro: IBGE; 2016 [acesso em 2018 nov 11]. Disponível em: https://biblioteca.ibge.gov.br/visualizacao/livros/liv97870.pdf

3. World Health Organization (WHO). Preventing suicide: a global imperative [Internet]. Genebra (Suíça): World Health Organization; 2014 [acesso em 2018 maio 12]. Disponível em: https://www.who.int/mental_health/suicide-prevention/world_report_2014/en/

4. Shrivastra SR, Shrivastava PS, Ramasamy J. Public health strategies to ensure reduction in suicide incidence in middle and low income nations. J Neurosci Rural Pract [Internet]. 2015 [acesso em 2018 maio 21];6(4):619-21. Disponível em: https:/www.ncbi.nlm.nih.gov/pmc/articles/PMC4692033/ 
5. World Health Organization (WHO). Disease burden and mortality estimates [Internet]. 2018 [acesso em 2019 maio 21]. Disponível em: https://www.who.int/healthinfo/global_burden_disease/estimates/en/index1.html

6. Waiselfisz JJ. Mapa da violência 2014: os jovens do Brasil [Internet]. Rio de Janeiro: FLACSO Brasil; 2014 [acesso em 2018 maio 21]. Disponível em: https://www.mapadaviolencia.org.br/pdf2014/Mapa2014_JovensBrasil_Preliminar.pdf

7. Conselho Federal de Psicologia (CFP). Prevenção ao suicídio: desafios para a psicologia e a saúde pública [Internet]. Brasília; 2017 [acesso em 2018 maio 21]. Disponível em: https://site.cfp.org.br/prevencao-ao-suicidio-desafios-para-psicologia-e-saude-publica/

8. Beserra MA. Violência na adolescência dentro do contexto escolar e fatores associados [tese]. Ribeirão Preto, Escola de Enfermagem de Ribeirão Preto: Universidade de São Paulo; 2015. 153 p.

9. Fialho V, Pires MJ, Neves RCM, Silva EP, Silva MMSG. Espaços compartilhados e práticas vividas: cartografia social e espaços de mobilização do bairro de Santo Amaro - Recife/PE. Iluminuras [Internet]. 2015 [acesso em 2018 nov 11];16(37):212-41. Disponível em: https://seer.ufrgs.br/iluminuras/article/view/53149

10. BRASIL. Presidência da República. Lei no 8.069, de 13 de julho de 1990. Dispõe sobre o Estatuto da Criança e do Adolescente e dá outras providências [Internet]. Brasília (DF); 1990 [acesso em 2019 abr 20]. Disponível em:http://www.planalto.gov.br/ccivil_03/leis/18069.htm

11. World Health Organization (WHO). Global school-based student health survey (GSHS) [Internet]. Genebra (Suíça): World Health Organization; 2012 [acesso em 2018 nov 11]. Disponível em: http://www.who.int/chp/gshs/en/

12. Freire IP, Simão AMV, Ferreira AS. O estudo da violência entre pares no 3ํㅗclo do ensino básico um questionário aferido para a população escolar portuguesa. Rev Port Educ [Internet]. 2006 [acesso em 2018 nov 11];19(2):157-83. Disponível em: http://www.scielo.mec.pt/scielo.php?script=sci_arttext\&pid=S0871-91872006000200008

13. Dalcin CB, Backes DS, Zanatta FB, Sousa FGM, Siqueira $\mathrm{HCH}$, Oliveira AMN. Fatores associados à violência em escolares: ampliando saberes e práticas para a enfermagem. Texto \& Contexto Enferm [Internet]. 2016 [acesso em 2018 nov 11];25(4):e4530014. Disponível em: http://www.scielo.br/pdf/tce/v25n4/pt_0104-0707-tce-25-04-4530014.pdf

14. Argaez Rodríguez SG, Echeverría Echeverría R, Evia Alamilla NM, Carrillo Trujillo CD. Prevención de factores de riesgo en adolescentes: intervención para padres y madres. Psicol Esc Educ [Internet]. 2018 [acesso em 2019 jan 11];22(2);259-69. Disponível em: https://dx.doi.org/10.1590/2175-35392018014279

15. Black DS, Grenard JL, Sussman S, Rohrbach LA. The influence of school-based natural mentoring relationships on school attachment and subsequent adolescent risk behaviors. Health Educ Res 
17 | Beserra MA, Souza SL, Silva MAI, Sena CA, Resende CMM, Ferriani MGC

[Internet]. 2010 [acesso em 2018 jan 11];25(5):892-902. Disponível em: https://www.ncbi.nlm.nih.gov/pubmed/20675354

16. Centers for Disease Control and Prevention (CDC). Understanding school violence [Internet]. Atlanta (EUA): Centers for Disease Control and Prevention; 2016 [acesso em 2018 jan 11]. Disponível em: https://www.cdc.gov/violenceprevention/pdf/School_Violence_Fact_Sheet-a.pdf

17. Carlos DM, Campeiz AB, Silva JL, Fernandes MID, Leitão MNC, Silva MAI, et al. School-based interventions for teen dating violence prevention: integrative literature review. Referência [Internet]. 2017 [acesso em 2018 jan 11];9(14):133-46. Disponível em: https://www.researchgate.net/publication/319996624_Schoolbased_interventions_for_teen_dating_violence_prevention_integrative_literature_review

18. Moynihan MM, Banyard VL, Cares AC, Potter SJ, Williams LM, Stapleton JG. Encouraging responses in sexual and relationship violence prevention: what program effects remain 1 year later? J Interpers Violence [Internet]. 2015 [acesso em 2018 maio 11];30(1):110-32. Disponível em: https://journals.sagepub.com/doi/10.1177/0886260514532719

19. Oliveira WA, Silva MAI, Silva JL, Mello FCM, Prado RR, Malta DC. Associações entre a prática de bullying e variáveis individuais e contextuais do ponto de vista dos agressores. J Pediatr (Rio J) [Internet]. 2016 [acesso em 2019 maio 30];92(1):32-9. Disponível em: http://www.scielo.br/scielo.php?script=sci_arttext\&pid=S0021$75572016000100032 \& \operatorname{lng}=\mathrm{en} \& \mathrm{nrm}=\mathrm{iso} \& \ln \mathrm{ln}=\mathrm{pt}$

20. Díaz-Aguado MJ. Da violência escolar à cooperação na sala de aula. Americana: Adonis; 2015. 440 p.

21. Gammone M, Perilli E, Recchioni M, Romualdo C, Sidoti F, Silva MAI. II contesto del suicidio. Trilhas Pedagóg [Internet]. 2016 [acesso em 2019 maio 30];6(6):257-87. Disponível em: http://www.fatece.edu.br/arquivos/arquivos\%20revistas/trilhas/volume6/16.pdf

22. Amare T, Meseret Woldeyhannes S, Haile K, Yeneabat T. Prevalence and associated factors of suicide ideation and attempt among adolescent high school students in Dangila Town, Northwest Ethiopia. Psychiatry J [Internet]. 2018 [acesso em 2019 maio 14];2018:7631453. Disponível em: https://www.hindawi.com/journals/psychiatry/2018/7631453/

23. Holt MK, Vivolo-Kantor J, Polanin JR, Holland KM, DeGue S, Matjasko JL, et al. Bullying and suicidal ideation and behaviours: a meta-analysis. Pediatrics [Internet]. 2015 [acesso em 2018 maio 14]; 135(2):e496-509. Disponível em: https://www.ncbi.nlm.nih.gov/pubmed/25560447

24- Kann L, McManus T, Harris WA, Shanklin SL, Flint KH, Queen B, et al. Youth risk behavior surveillance - United States, 2017. MMWR Surveill Summ [Internet]. 2018 [acesso em 2019 maio 30]; 2018;67(8):1-114. Disponível em: https:/www.cdc.gov/mmwr/volumes/67/ss/ss6708a1.htm 
25. Silva KL, Sena RR, Gandra EC, Matos JAV, Coura KRA. Promoção da saúde no Programa Saúde na Escola e a inserção da Enfermagem. REME Rev Min Enferm [Internet]. 2014 jul-set [acesso em 2018 maio 14];18(3):614-22. Disponível em: http://www.reme.org.br/artigo/detalhes/950

\section{Autor correspondente}

Maria Aparecida Beserra

E-mail: aparecida.beserra@upe.br

Endereço: Faculdade de Enfermagem Nossa Senhora das Graças - Universidade de Pernambuco

Rua: Dr. Otávio Coutinho - Bairro Santo Amaro, Recife - Pernambuco, Brasil

CEP: 52171 - 011

\section{Contribuições de Autoria}

\section{1 - Maria Aparecida Beserra}

Participou da concepção e planejamento do projeto de pesquisa; obtenção, análise e interpretação dos dados; redação e revisão crítica.

\section{2 - Sinara de Lima Souza}

Revisão crítica.

\section{3 - Marta Angélica Iossi Silva}

Revisão crítica.

\section{4 - Cláudia Alves de Sena}

Revisão crítica.

\section{5 - Cristina Maria Mendes Resende}

Revisão crítica.

\section{4 - Maria das Graças Carvalho Ferriani}

Participou concepção e planejamento do projeto de pesquisa; análise e interpretação dos dados e revisão crítica.

\section{Agradecimentos}

À Fundação de Amparo de Ciência e Tecnologia do Estado de Pernambuco (FACEPE) e ao Conselho Nacional de Desenvolvimento Científico e Tecnológico (CNPq) pela bolsa de fomento ao pesquisador principal.

\section{Como citar este artigo}

Beserra MA, Souza SL, Silva MAI, Sena CA, Resende CMM, Ferriani MGC. Violência no contexto escolar e ideação suicida na adolescência. Rev. Enferm. UFSM. 2020 [Acesso em: Anos Mês Dia]; vol.10 e71: 1-18. DOI:https://doi.org/10.5902/2179769238005 